\title{
Modelo mejorado de velocidades unidimensional de corteza y manto superior en el transarco andino argentino entre latitudes $31.5^{\circ}$ y $33.5^{\circ} \mathrm{S}$
}

\author{
Renzo Furlani* \\ Enrique G. Triep \\ Silvina Nacif Suvire
}

Recibido en agosto de 2014; aceptado en enero de 2015

\begin{abstract}
Using a one-dimensional approach of the earth, a new P-wave velocity model (Vp) was determined for crust and upper mantle that improve hypocenter localization in the South-Central Andean foreland of Argentina. This region that shows intense crustal and deep earthquake seismic activity have been monitoring by appropriate seismic local network during a period of 3 month. A total of 12,841 first arrival Pwave were identified. This data set was produced by 1,262 intermediate deep earthquake and 449 crustal earthquakes. Considering a pre-defined Vp model, a subset of well constrained earthquakes were selected as input data for inversion process. Tree layer crustal and one layer upper mantle Vp model was adopted. VELEST code was used for simultaneous inversion of velocity, hypocenter and station correction parameters. Results for different damping parameters were evaluated at the end of process inversion. The final crustal model shows Vp of $5.95,6.15$ y $6.82 \mathrm{~km} / \mathrm{s}$ related to deep interfaces of 10, 32 and $45 \mathrm{~km}$ below sea level. Root mean square (RMS) of residuals was $0.31 \mathrm{~s}, 20.5 \%$ less than initial localizations. Vp for upper and middle crust layer exhibit light changes related to the initial model. For upper crust, the final Vp was slightly bigger than the initial model suggesting a more participation of crystalline basement rock. In lower crust, resulting Vp was less than expected for determinations at north of our network suggesting a possible rock composition segmentation. Vp for upper mantle of $7.34 \mathrm{~km} / \mathrm{s}$ was very lower than previous works possible effect of poor conditioning. This Vp was finally defined as $8.04 \mathrm{~km} / \mathrm{s}$. Stations corrections result keep inside normal limits and they are consistent with de-
\end{abstract}

* Instituto Geofísico Sismológico F.S. Volponi, Universidad Nacional de San Juan, Ruta 12, km. 17, Jardín de los Poetas, C.P. 5407, Marquesado, San Juan Argentina, correo electrónico: rfurlani1@gmail.com 
terminations using data from other seismology network. The general pattern of seismicity has not strongly changed but more precise localizations could be able to illuminate new features and more reliable correlations with some tectonics and structural domains. The new velocity model here proposed reduces hypocenter localization uncertainty and it is defined as a new Vp local model for crust and upper mantle for precise and fast localization of crustal and intermediate deep earthquakes in the region where, Nazca plate is in flat subduction and transition to normal and also concentrates the most earthquake activity of Argentina.

Key words: One-dimensional velocity model, hypocenter determination, Argentinian Andean foreland, residues, crust, upper mantle.

\section{Resumen}

Mediante el uso de una aproximación unidimensional (1D) estratificada de la tierra, se determinó un nuevo modelo de velocidad de onda $\mathrm{P}(\mathrm{Vp})$ de manto y corteza superior que mejora la localización de sismos en el antepaís andino argentino. Esta región presenta una intensa actividad sísmica cortical y de profundidad intermedia. Durante un periodo de tres meses, una apropiada red local de estaciones sismológicas permitió identificar 12,841 primeros arribos de onda $\mathrm{P}$ correspondientes a 1,262 sismos de profundidad intermedia y 449 sismos de corteza. Teniendo en cuenta un modelo de Vp predefinido, se seleccionó un subconjunto de sismos bien condicionados como datos de entrada para el proceso de inversión. El modelo inicial consistió en tres capas de velocidades para corteza y una para manto superior. El código VELEST fue utilizado para la determinación simultánea de velocidades, localización de hipocentros y corrección de estación. Se evaluó el desempeño del algoritmo y sus resultados finales considerando diferentes parámetros de amortiguamiento. El modelo de corteza obtenido presentó velocidades de $5.95,6.15$ y $6.82 \mathrm{~km} / \mathrm{s}$ correspondientes a profundidades de interface de 10,32 y $45 \mathrm{~km}$ debajo del nivel del mar. La raíz cuadrada de las diferencias cuadráticas (RMS) de residuos fue de $0.31 \mathrm{~s}$, $20.5 \%$ menor que la obtenida con modelo de Vp inicial. La velocidad de onda $\mathrm{P}$ para corteza superior fue levemente más alta sugiriendo mayor participación en esta zona de rocas de basamento cristalino. En la corteza inferior la velocidad obtenida es menor a las evidencias sismológicas principalmente al norte de la red, lo que sugiere una posible segmentación de composición de corteza inferior. La velocidad de manto superior con una determinación de $7.34 \mathrm{~km} / \mathrm{s}$ resultó muy baja sugiriendo un pobre condicionamiento por lo que fue definida a $8.04 \mathrm{~km} / \mathrm{s}$. Las correcciones de estación se mantienen en general dentro de rangos normales y consistentes con determinaciones obtenidas a partir de datos de otra red sismológica. El patrón general de sismicidad no ha cambiado drásticamente pero las localizaciones más precisas podrán ser capaces de iluminar nuevas evidencias y más confiables 
correlaciones con las características tectónicas y estructurales de la región. El modelo de velocidades de onda $\mathrm{P}$ aquí propuesto reduce la incertidumbre en la localización de hipocentros y se propone como un nuevo modelo local de corteza y manto superior para la rápida y preliminar localización de sismos de profundidad intermedia y corteza dentro del área por encima de placa de Nazca en transición de subducción plana a normal donde se concentra la mayor actividad sísmica cortical de Argentina.

Palabras clave: Modelo unidimensional de velocidades, determinación de hipocentros, transarco andino argentino, residuos, corteza, manto superior.

\section{Introducción}

Los modelos de velocidades han sido extensivamente utilizados para conocer la estructura geológica, composición y estado físico de rocas. Los modelos pueden ser construidos a partir de ciertas observaciones obtenidas de registros de un sismo por ejemplo, su forma de onda o su tiempo de viaje. En la zona del antepaís andino argentino (Figura 1), los modelos de velocidades de onda $\mathrm{P}(\mathrm{Vp})$ de corteza y manto superior entre latitudes 31 a $33.5^{\circ} \mathrm{S}$ no han sido obtenidos simultáneamente a partir de tiempos de viaje de ondas P. Por lo contrario, algunas determinaciones de Vp han sido obtenidas mediante el procesamiento de formas de onda $\mathrm{P}$ telesísmicas y regionales. Así, un modelo de $\mathrm{Vp}$ de corteza ha sido inferido mediante análisis de sensibilidad de velocidades a la inversión de forma de onda de tensor momento (Alvarado et al., 2007). Como resultado, se obtuvieron velocidades promedio de corteza a escala regional con una menor resolución al sur de $31.5^{\circ} \mathrm{S}$ que al norte. La forma de onda de sismos locales fue utilizada para obtener funciones de receptor de estación única infiriendo un modelo de velocidad de onda S (Vs) (Calckins et al., 2006). Si bien otro estudio local permitió conocer en detalle la sismicidad de corteza (ej. Smalley et al., 1993; Pujol et al., 1992), los tiempos de viaje de estos sismos locales se utilizaron para construir un modelo de velocidad únicamente de corteza media (Bollinguer y Langer, 1981). Aún más, estos autores no han considerado este conjunto de datos para obtener un modelo de Vp simultáneamente tanto para niveles de corteza más someros como para niveles más profundos que involucren el manto superior. Otro estudio determinó localizaciones regionales de sismos de placa considerando modelos de $\mathrm{Vp}$ regional unidimensional para corteza y manto superior (Anderson et al., 2007), pero esta información no ha sido utilizada para reajustar dicho modelos. Modelos tomográficos tridimensionales de velocidad de manto superior fueron obtenidos a partir de sismos de profundidad intermedia registrados por la red regional CHARGE (Wagner et al., 2005). Estos autores indicaron que los sismos corticales observados no se usaron para resolver velocidades corticales debido a la imprecisión en la lectura de tiempos de arribo de fases Pg y Sg por 
contaminación de la coda de los arribos más tempranos de fases Pn y Sn. Por ende, dichas velocidades de manto superior no han podido determinarse simultáneamente con las correspondientes de corteza. Por último, el experimento sismológico SIEMBRA (Universidad de Arizona, Estados Unidos; Instituto Nacional de Prevención Sísmica, Universidad Nacional de San Juan, Argentina) desplegó una red local de estaciones temporarias que permitió registrar la sismicidad cortical y de profundidad intermedia que permitiría obtener modelos de velocidades de corteza y manto superior a partir de inversión de tiempos de viaje de sismos. La red de estaciones comprendió principalmente una franja entre 31 a $32^{\circ} \mathrm{S}$. Los resultados aquí obtenidos podrán complementarse con los de SIEMBRA debido a que corresponden a una red localizada inmediatamente al sur.

En la localización típica de sismos los parámetros de velocidad permanecen fijos a valores definidos a priori, que se asumen como correctos. No considerar el acoplamiento entre los parámetros de velocidades e hipocentros durante el proceso de localización puede conducir a errores sistemáticos en la determinación de hipocentros (Thurber, 1992, Eberhart-Phillips y Michael, 1993). Aún más, la estimación de errores depende decisivamente del modelo de velocidad asumido (Kissling et al., 1994). Ambas consideraciones ponen de manifiesto la importancia de la resolución conjunta de parámetros de velocidades e hipocentrales. Si bien las velocidades de manto superior o de corteza media han sido determinadas a partir de tiempos de viaje de sismos (Wagner et al., 2005; Bollinguer y Langer, 1988), estas no lo han sido así conjuntamente, y sólo se han obtenido en forma independiente por distintos autores. Por un lado no existen modelos de Vp que sean obtenidos simultáneamente para toda la corteza como el manto superior. Además, tampoco han sido basados en tiempos de viaje tanto de sismos corticales como de sismos a profundidad intermedia. Los modelos unidimensionales (1D) constituyen una implementación práctica del medio para resolver conjuntamente por velocidades e hipocentros.

La región del transarco andino argentino entre 31 a $33.5^{\circ} \mathrm{S}$ presenta una configuración tectónica con una placa superior sobremontada a otra placa inferior subducida de forma plana. La placa continental Sudamericana está constituida por un mosaico de terrenos acrecionados (Figura 2) durante el Proterozoico tardío y Paleozoico (Rapela et al., 1998; Ramos, 2009; Aceñolaza y Toselli, 2002; Ramos, 1986) donde la mayor actividad sísmica se concentra dentro del terreno Cuyania (Alvarado et al., 2007). Por debajo subduce la placa oceánica de Nazca con un estilo subhorizontal (Cahill y Isaacks, 1992; Anderson et al., 2007) siendo presumiblemente responsable de la actividad sísmica cortical (Gutscher, 2002). Una intensa actividad sísmica asociada a los esfuerzos presentes en la placa subducida plana y su transición a subducción normal al sur desarrolla sismos de profundidad intermedia (Anderson et al., 2007). A pesar de esta actividad sísmica registrada en ambos niveles de profundidad, tanto los sismos corticales como los de profundidad intermedia no 
han sido utilizados para obtener un modelo de velocidades de corteza y manto superior que permita una más precisa localización de hipocentros.

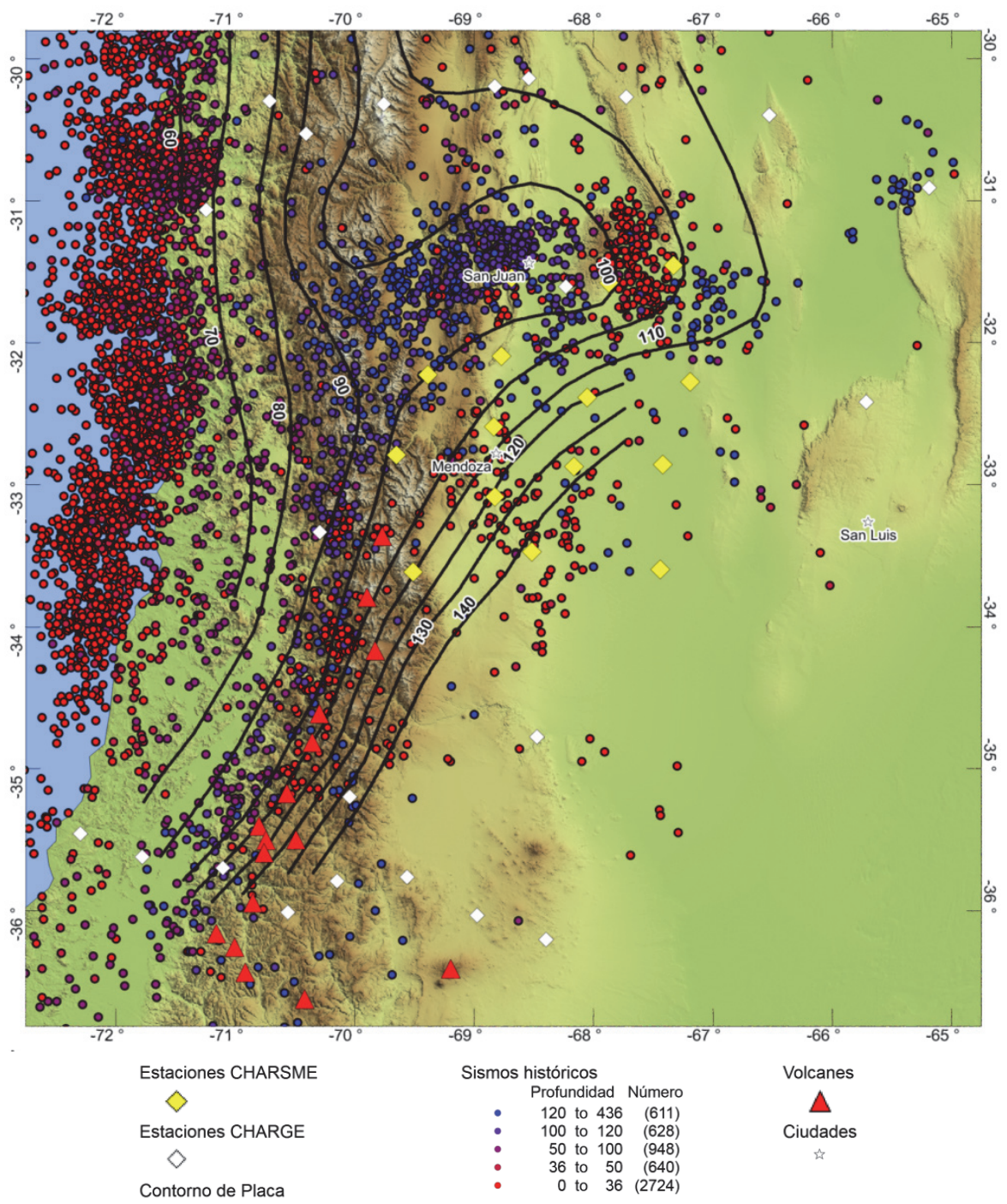

Figura 1. Mapa de localización de la región de estudio. Red sismológica temporal regional CHARGE y red temporal local CHARME. Líneas de contorno de placa subducida de Nazca por Anderson et al., 2007. Sismicidad histórica obtenida del catálogo internacional de sismos NEIC con magnitud $>5$. Volcanismo cuaternario de arco por Stern, 2004. 


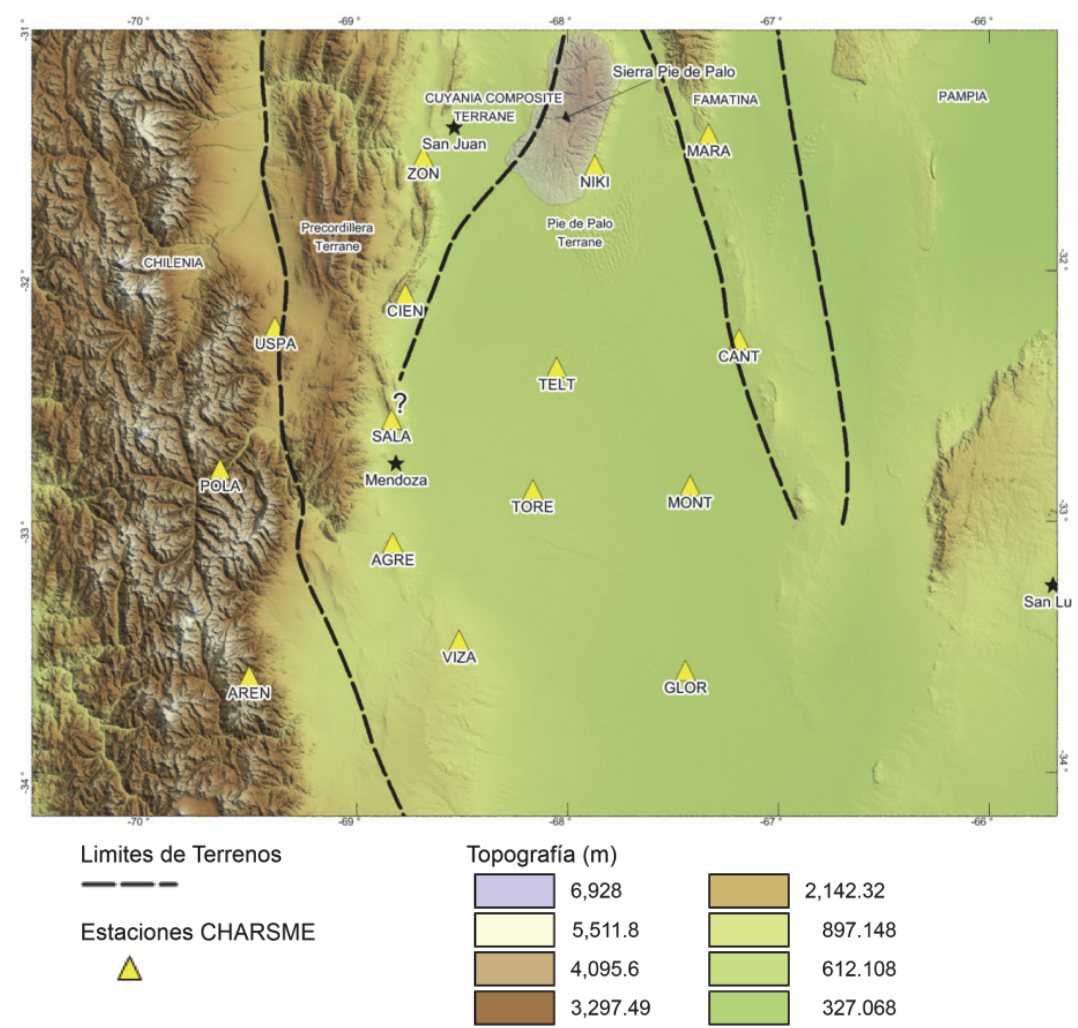

Figura 2. Red local de estaciones sismológicas CHARSME y topografía. Suturas y terrenos de Ramos et al., 2002, Ramos, 2004.

En este trabajo se siguió el procedimiento de Kissling (1998) y Kissling et al. (1994) que utiliza un algoritmo de inversión de modelo de velocidad 1D para la resolución simultánea iterativa de inversión de hipocentros y modelo de velocidades. El modelo será obtenido a partir de datos de lecturas de primeros arribos de onda $\mathrm{P}$ relacionados a sismos de corteza y profundidad intermedia comprendidos dentro de una red local. Este modelo será óptimo en el sentido que minimizará el RMS de residuos mediante el ajuste simultáneo de los parámetros de velocidad, corrección de estación y relocalización de hipocentros. Además, será el primer modelo de Vp unidimensional que comprende la estructura de corteza y de manto superior obtenido únicamente usando los tiempos de viaje de onda P. Este modelo será más adecuado para la región y permitirá una rápida y más precisa localización de sismos corticales y profundidad intermedia que podrá proporcionar nuevos y más detalles de la sismicidad de corteza y a profundidad intermedia en el antepaís andino argentino. 


\section{Red de estaciones y datos}

El experimento sismológico CHARSME (CHile ARgentina SeisMic Experiment; Universidad Nacional de San Juan de Argentina, Universidad de Chile, Géoazur de Francia), desplegó estaciones sismológicas banda ancha en Chile y Argentina, durante un periodo de tres meses comprendido entre noviembre de 2002 hasta febrero del 2003. En este trabajo se usaron solamente la subred de 13 estaciones distribuidas en terreno argentino y se incorporó la estación permanente ZON (Instituto Geofísico Sismológico F.S. Volponi, Universidad Nacional de San Juan). Las estaciones fueron distribuidas principalmente dentro del Terreno Cuyania y menor cantidad de ellas dentro de los terrenos adyacentes de Pampia y Chilenia (Figura 2). Las estaciones temporarias tuvieron registradores de 24 bits TITAN (ARGEODAIS). El área cubierta dentro de Argentina fue de aproximadamente 250 por $250 \mathrm{~km}$ con una distancia entre estaciones de $50-70 \mathrm{~km}$.

La lectura de primeros arribos de onda $\mathrm{P}$ fue obtenida por inspección visual de la componente vertical. Las fases fueron clasificadas como impulsivas o emergentes. Se asignaron pesos dependiendo de la incertidumbre en la determinación del tiempo de arribo (Tabla 1). En lo posible, las determinaciones se realizaron sin filtrar la señal, sin embargo, en algunos casos de pobre identificación se aplicaron filtros pasa banda a-causales con frecuencias de paso de $1-10 \mathrm{~Hz}$ y $1-15 \mathrm{~Hz}$ que atenuaron el ruido fundamentalmente generado por el microsismo oceánico. Se reconocieron señales precursoras de baja amplitud $\sim 0.25$ segundos antes de aparecer un evento altamente impulsivo. Este fué un efecto del filtro FIR a-causal del conversor analógico-digital (ADC) de los registradores (ej., Wagner et al., 2005). Se inspeccionaron cuidadosamente estas fases para realizar las determinaciones justo al comienzo de la onda impulsiva y no al comienzo de la señal precursora artificial. Los arribos de fases directas Pg fueron cuidadosamente determinados e identificados en sismos suficientemente lejanos a la estación que podrían confundirse con fases de onda Pn adelantadas en el tiempo.

Tabla 1

Asignación de pesos a fases $\mathbf{P}$

\begin{tabular}{cc}
\hline Incertidumbre, $s$ & Peso \\
\hline 0.05 & 1 \\
0.10 & $0.75(3 / 4)$ \\
0.15 & $0.50(1 / 2)$ \\
0.20 & $0.25(1 / 4)$ \\
$>0.25$ & 0 \\
\hline
\end{tabular}


Como último control de calidad de datos se investigaron las lecturas que correspondían a considerables residuos. Previo a esto, todos los sismos detectados fueron inicialmente localizados usando el modelo de velocidad de Smalley et al., (1993) y el software de localización HYPO2000 (Klein, 1999). Se inspeccionaron lecturas con diferencias absolutas entre tiempo de viaje predicho y observado (residuo) por encima de $1 \mathrm{~s}$. Se detectaron un total de 1,262 sismos de placa y 449 sismos de corteza.

\section{Procesamiento}

Se usó el software VELEST (Kissling, 1994; Kissling et al., 1995) que implementa una solución linealizada iterativa del problema acoplado de hipocentros-modelo de velocidad. Este código resuelve conjuntamente la determinación de hipocentros, el ajuste del modelo de velocidad unidimensional y las correcciones de estación mediante la minimización local de RMS de residuos (raíz cuadrática media de la diferencia de tiempos de viaje observados con los predichos) de un conjunto de sismos seleccionados. Si se mantiene fijo el modelo de velocidad a lo largo de todo el proceso de inversión, VELEST implementa la relativa localización conjunta de hipocentros (Joint Hypocenter Determination, JHD) (ej. Pujol et al., 1992). Este código necesita, además de los tiempos de primeros arribos, cuatro conjuntos de datos adicionales que consisten en: un modelo inicial de velocidades, localizaciones iniciales, las coordenadas de localización de estaciones y un conjunto de parámetros de inversión.

El modelo de Vp para la corteza y el manto superior seleccionado como inicial fue uno utilizado en investigaciones anteriores (ej. Smalley et al., 1993; Pujol et al., 1992). Su sección cortical presenta tres capas de velocidades para modelar la corteza. Cada capa correspondiente a corteza superior, media e inferior presenta un espesor de 10, 22 y $13 \mathrm{~km}$ respectivamente (Tabla 2). Este modelo cortical considera velocidades que han sido obtenidas mediante estudios geofísicos independientes, como ser de refracción sísmica petrolera y a partir de datos sismológicos de red temporaria de estaciones utilizadas para registro de réplicas (Bollinguer y Langer, 1988). El espesor total de corteza de $45 \mathrm{~km}$ se encuentra en consistencia con los espesores corticales usados para localizaciones basadas en redes regionales (Anderson et al., 2007) o bien representa la profundidad promedio entre las determinaciones de profundidad de Moho variable de $\sim 40 \mathrm{~km}$ sobre el borde Este de la red a valores de $\sim 60 \mathrm{~km}$ al borde Oeste en Cordillera Frontal (Alvarado et al., 2007; Gilbert et al., 2006). La velocidad inicial para el manto superior de $8.1 \mathrm{~km} / \mathrm{s}$, que también constituye la asignada para el semiespacio inferior, fue obtenida mediante otro estudio sismológico independiente basado en ondas telesísmicas (Volponi, 1968). No obstante otros modelos de Vp más recientes han sido determinados y considera- 
dos (Anderson et al., 2007; Alvarado et al., 2007) y que alguno de ellos podría considerarse como modelo inicial, no fueron elegidos porque consisten una a dos capas para el modelo de corteza y una capa de velocidad para el manto superior. Se decidió por uno más detallado con cuatro capas.

Tabla 2

Modelo de Vp de tres capas y subespacio inferior

\begin{tabular}{ccc}
\hline Velocidad $\mathrm{km} / \mathrm{s}$ & Profundidad $\mathrm{km}$ & Espesor $\mathrm{km}$ \\
\hline 5.88 & 0.0 & 10.0 \\
6.20 & 10.0 & 22.0 \\
7.30 & 32.0 & 13.0 \\
8.10 & 45.0 & $\infty$ \\
\hline
\end{tabular}

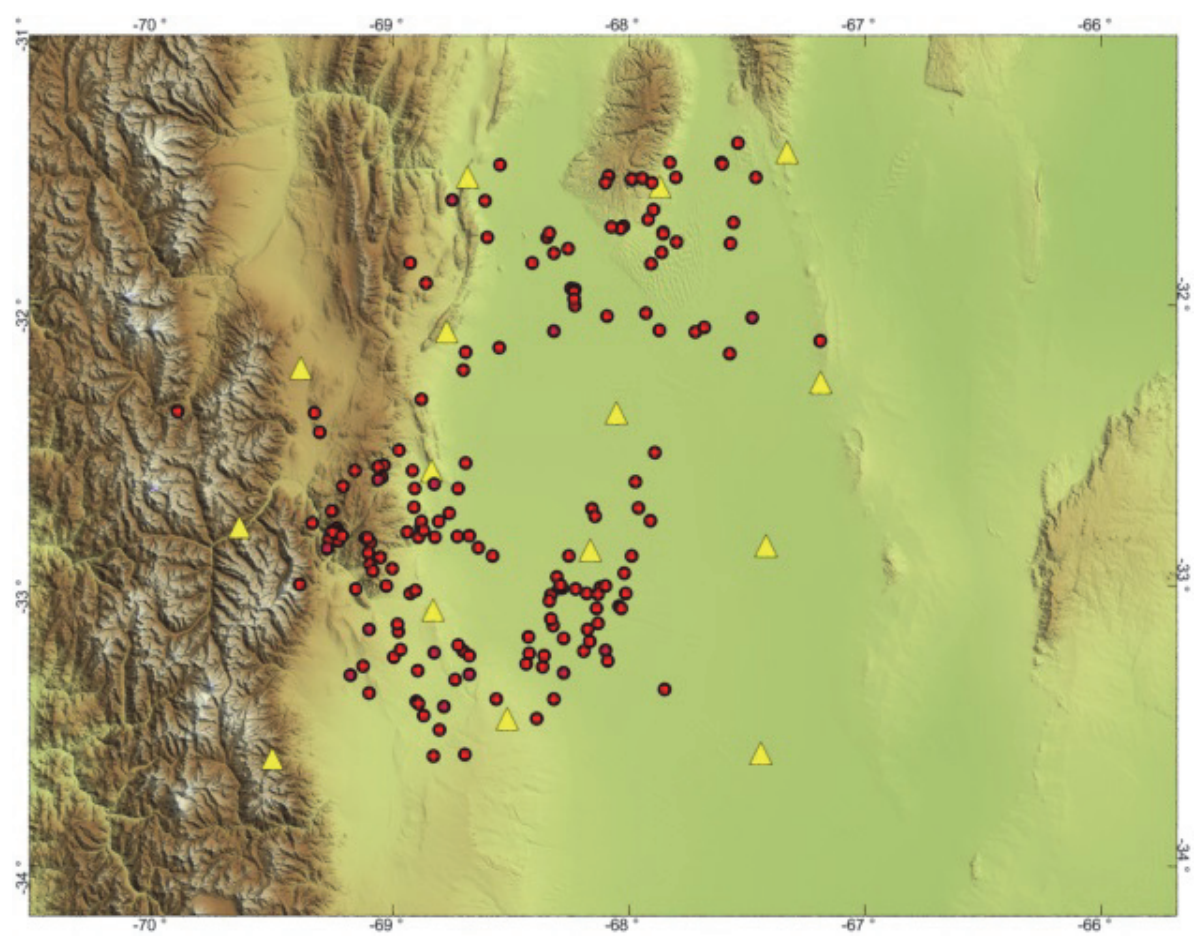

Figura 3. Sismos de corteza involucrados dentro del proceso de inversión. 


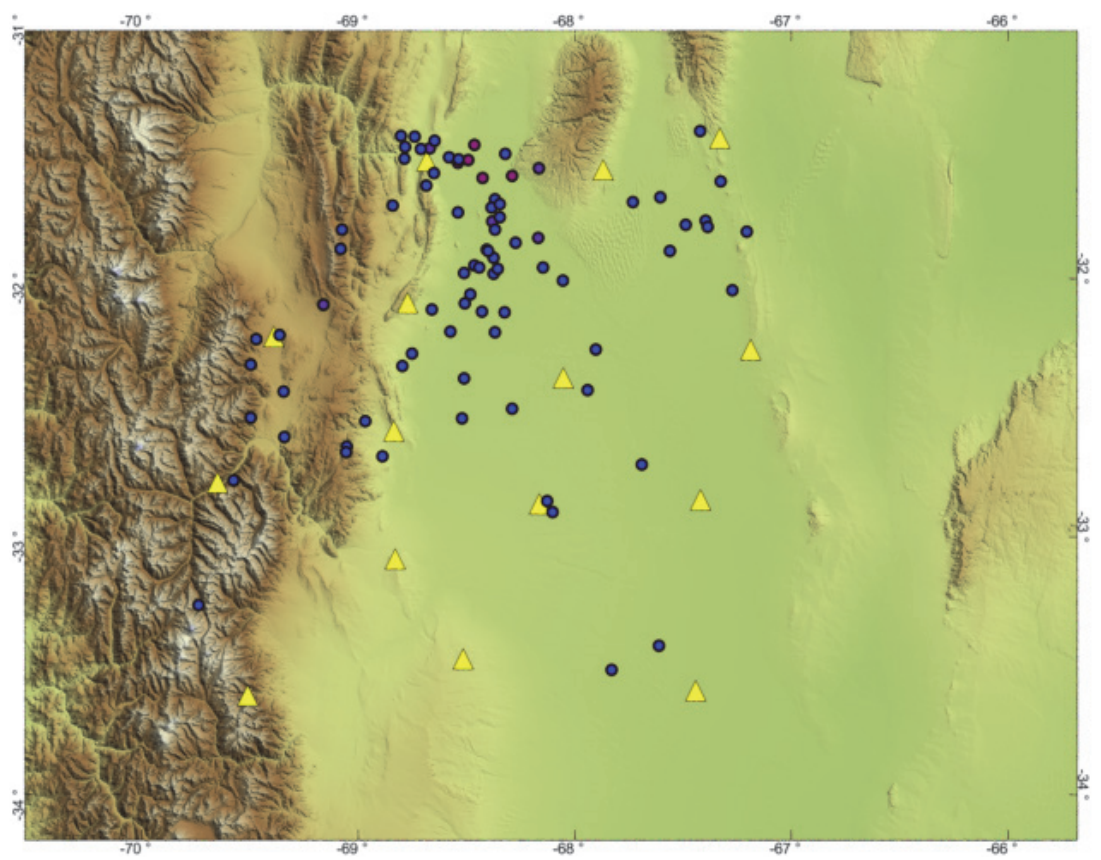

Figura 4. Sismos de profundidad intermedia involucrados dentro del proceso de inversión.

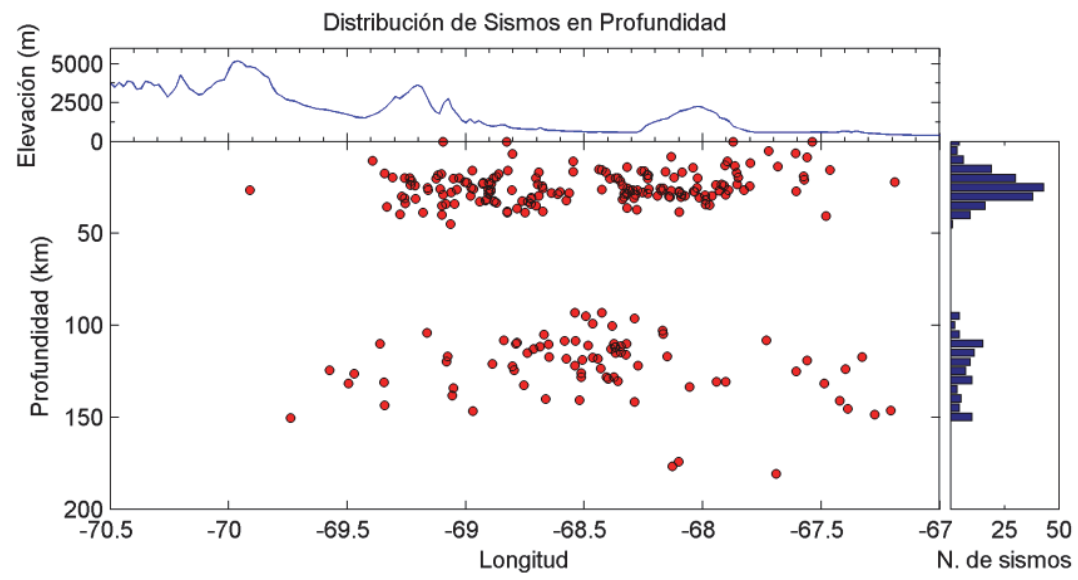

Figura 5. Distribución de sismos en profundidad involucrados dentro del proceso de inversión. 
Según Kissling et al., 1994, es necesario seleccionar un número de sismos de alta calidad y precisa localización para la construcción de un modelo de velocidad. Así, se seleccionaron un conjunto de sismos de corteza y de profundidad intermedia cuya cobertura acimutal de estaciones sea mayor a $180^{\circ}$ y un número de lecturas de onda P $>8$. Considerando las localizaciones obtenidas usando HYPO2000 (Klein, 2002) como localizaciones iniciales, se seleccionaron un total de 221 sismos de corteza con 1,861 lecturas de onda P y 72 sismos de placa presentan 648 lecturas de onda $\mathrm{P}$.

Los sismos corticales seleccionados se encuentran concentrados principalmente en diversos grupos relativamente bien separados entre sí (Figura 4). Los sismos de profundidad intermedia se concentran principalmente en el cuadrante noroeste de la red y tienden a distanciarse cada vez más entre sí a medida que se localizan más al sur y al este (Figura 4). Estos ocurren donde la placa de Nazca de geometría subhorizontal se flexiona para acomodarse a una subducción normal hacia el sur. El histograma en profundidad de los sismos seleccionados muestra una distribución bimodal. Los sismos corticales están comprendidos principalmente entre 15 a $45 \mathrm{~km}$ de profundidad. Llamativamente, las localizaciones iniciales presentan sistemáticamente algunos sismos corticales $(>5)$ a profundidad cero. Este fenómeno no sugiere la evidencia de sismos a niveles muy someros, si no presumiblemente inadecuadas velocidades del modelo de $\mathrm{Vp}$ que no pueden resolver satisfactoriamente los tiempos de arribo observados. La mayoría de los sismos de placa involucrados se encuentran concentrados entre los 90 a $130 \mathrm{~km}$ de profundidad. Son escasos los sismos por debajo de $140 \mathrm{~km}$. Así el modelo de Vp obtenido comprenderá hasta los $120 \mathrm{~km}$ de profundidad. No se han determinado sismos entre 50 a $100 \mathrm{~km}$ (Figura 5).

Los parámetros del algoritmo que controlan la evolución del proceso de inversión deben ser ajustados para obtener resultados adecuados. Los parámetros de amortiguamiento controlan la velocidad, estabilidad de convergencia y la participación relativa de las distintas incógnitas involucradas. VELEST permite asignar amortiguamientos específicos para las determinaciones de velocidades, correcciones de estación y relocalización de sismos. El peso relativo de estos tres parámetros determinará la influencia relativa de cada conjunto de incógnitas en la minimización progresiva del indicador de ajuste RMS de residuos. Se probaron diversas combinaciones de estos para evaluar sus valores y su impacto en la obtención de un nuevo modelo de Vp.

VELEST impone que las estaciones sismológicas en zonas de topografía variable se encuentren comprendidas dentro de la primera capa. En casos de extrema excursión topográfica la primera capa adquiere un espesor inapropiado produciendo inestabilidades en la determinación de $\mathrm{Vp}$ de las primeras capas (Husen et al., 2002). Este autor ha resuelto ese problema eliminando la elevación topográfica de 
las estaciones, consecuentemente las correcciones de estación representan en parte el efecto topográfico no considerado sobre tiempo de viaje de la onda sísmica. $A$ priori no se usó esta solución pero es necesario conocer si el algoritmo de inversión convergió a un resultado consistente. Así, se evaluó el resultado final de la primera capa y los rangos de correcciones de estación en relación a cierto conocimiento estructural y antecedentes sismológicos.

Se seleccionó AGRE como estación de referencia ya que presenta un gran número de lecturas y cobertura acimutal completa de rayos y se encuentra sobre la bien explorada zona petrolífera de Cuenca Cuyana. Las estaciones más cercanas al centro geométrico de la red no fueron seleccionadas debido a que se encuentran sobre una cobertura sedimentaria neógena denominada Llanura Nororiental Mendocina de escasos estudios geológicos.

No se dio la posibilidad de resolver por capas de baja velocidad ya que no hay antecedentes sismológicos de zonas de baja velocidad. Aun así, se debe considerar la posibilidad de que existen este tipo de anomalías. Una posible causa de zonas debajo velocidad es producto de material fundido emplazado en corteza (Ji et al., 2009). Sin embargo en la región de estudio el arco volcánico activo ha migrado hacia el cratón en relación al aplanamiento progresivo de placa subducida (Gutscher, 2002; Ramos et al., 2002; Kay et al., 2002). La reaparición del arco volcánico activo a partir de $33.5^{\circ} \mathrm{S}$ (Hildreth y Moorbath, 1988; Stern, 2004) se encuentra fuera del área de estudio.

No se utilizaron como velocidades iniciales de manto superior aquellas determinadas para la zona de estudio por Wagner et al., 2005. Se utilizó la velocidad inicial de $8.1 \mathrm{~km} / \mathrm{s}$ del modelo de Smalley et al., 1993 , muy cercana al valor de $8.04 \mathrm{~km} / \mathrm{s}$ utilizada como inicial por esta autora para la construcción de sus modelos tomográficos. Tampoco se utilizaron las determinaciones de profundidad de la Moho dentro del terreno Cuyania (Perarnau et al., 2010; McGlashan et al., 2009; Heit et al., 2008; Alvarado et al., 2007; Calckins et al., 2006; Gilbert et al., 2006) que han propuesto una Moho de topografía variable basándose en observaciones sismológicas principalmente comprendidas por arriba de la placa plana y al norte del área de estudio. Lamentablemente, VELEST no determina las profundidades de cada capa. Por la incertidumbre en definir una Moho promedio se adoptó la profundidad del modelo inicial de $45 \mathrm{~km}$, un valor razonable como se discutió en párrafos anteriores.

\section{Resultados y discusión}

\section{Parámetros de inversión y desempeño}

El valor de amortiguamiento de localización de hipocentros de 0.01 junto a un amortiguamiento de correcciones de estación de 80 y de velocidades de 40 en gene- 
ral condujeron a valores de RMS relativamente pequeños. Los amortiguamientos de velocidades por debajo de 40 tendieron a inestabilizar el algoritmo. Los amortiguamientos por encima aumentaron los retardos de estación a valores absolutos de hasta de $\sim 1 \mathrm{~s}$. Estas determinaciones resultaron posiblemente irreales si se considera que duplicaron la media del valor absoluto de las correcciones de estación obtenidas por Pujol et al., 1992. En este escenario, el aumento del amortiguamiento de velocidades y las correcciones de estación al mismo tiempo tendieron a aumentar el RMS. Los amortiguamientos de velocidades en la franja de 50 a 80 , junto con un amortiguamiento de estaciones de $10 \mathrm{y}$ un amortiguamiento de hipocentros de 0.01 lograron reducidos RMSs, estabilizaron la convergencia y determinaron retardos de estación con valores dentro de 0.5 a $-0.5 \mathrm{~s}$. Las velocidades obtenidas para amortiguamientos de $\mathrm{Vp}$ dentro de esta franja permanecieron prácticamente idénticas. De la misma manera, los amortiguamientos de estación comprendidos entre 1 a 80 presentaron poca sensibilidad sobre las determinaciones de correcciones de estación. Se seleccionó un valor de 60 para amortiguamiento de velocidad (Tabla 3).

Tabla 3

Parámetros de inversión

\begin{tabular}{lc}
\hline \multicolumn{1}{c}{ Parámetro de inversión } & Valor \\
\hline Número de iteraciones & 10 \\
Mínimo cambio de RMS permitido & $0.05 \mathrm{~s}$ \\
Ajuste máximo de velocidad & $0.2 \mathrm{~km} / \mathrm{s}$ \\
Amortiguamiento de velocidad & 60.0 \\
Ajuste máximo de profundidad & $5.0 \mathrm{~km}$ \\
Amortiguamiento lateral de hipocentros $(\mathrm{x}, \mathrm{y})$ & 0.01 \\
Amortiguamiento de tiempo origen & 0.01 \\
Amortiguamiento de corrección de estación $(\mathrm{x}, \mathrm{y})$ & 10.0 \\
Amortiguamiento de profundidad & 0.01 \\
\hline
\end{tabular}

Las localizaciones iniciales basadas en el modelo inicial presentaron un RMS de $0.39 \mathrm{~s}$. El nuevo modelo de $\mathrm{Vp}$ junto a correcciones de estación indicó un RMS de $0.311 \mathrm{~s}$. Esta disminución implica menores errores de localización con respecto a aquellas iniciales.

Considerando el modelo inicial y su distribución de velocidades en profundidad, el modelo de $\mathrm{Vp}$ de corteza y manto superior aquí obtenido es uno de distribución de capas en profundidad idéntica, pero más adecuado y representativo para la zona del transarco andino argentino entre 31 y $33.5^{\circ} \mathrm{S}$. Al contrario de modelos anteriores, este ha sido obtenido mediante la inversión de primeros arribos de lecturas de onda $\mathrm{P}$, asociadas a los tiempos de viaje de rayos provenientes de sismos de corteza y placa subducida. 
Por otro lado, los resultados del modelo unidimensional final de Vp y las correcciones de estación se vinculan con ciertas heterogeneidades de $\mathrm{Vp}$ relacionadas a consideraciones geológicas determinadas. Como cualquier problema de inversión, las soluciones obtenidas no son únicas, lo que sugiere que diversos modelos de velocidades podrían ser los obtenidos a partir de diferentes conjuntos de datos y parámetros de algoritmo. Además, el código utilizado resuelve por minimización lineal de residuos lo que implica que otros mínimos locales pueden satisfacer la búsqueda iterativa óptima de parámetros de velocidades, hipocentros y corrección de estación (Kissling et al., 1994). Cabe señalar y tener presente que ciertas determinaciones son más precisas que otras, lo que conduce a un sistema de ecuaciones de condicionamiento mixto.

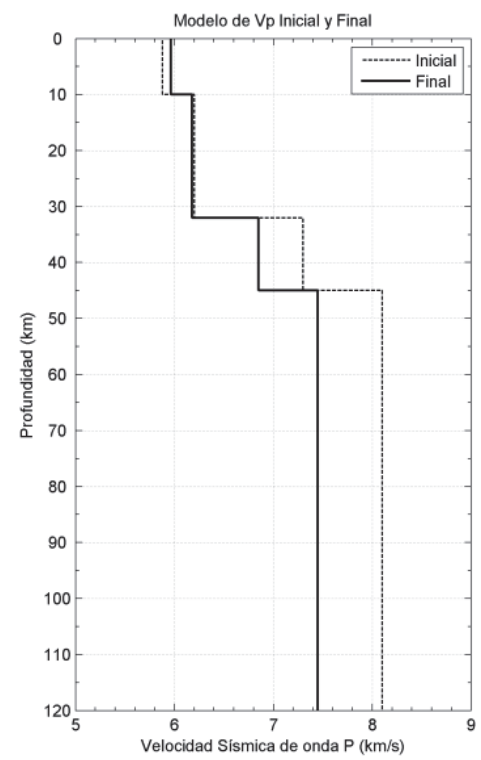

Figura 6. Modelo inicial de Smalley et al., 1993 y modelo final obtenido luego del proceso de inversión.

Tabla 4 Modelo inicial y final de Vp de tres capas y subespacio inferior

\begin{tabular}{ccc}
\hline Profundidad, $\mathrm{km}$ & Vp de modelo inicial, $\mathrm{km} / \mathrm{s}$ & Vp de modelo Final, $\mathrm{km} / \mathrm{s}$ \\
\hline 0.0 & 5.88 & 5.95 \\
10.0 & 6.20 & 6.15 \\
32.0 & 7.30 & 6.80 \\
45.0 & 8.10 & 7.35 \\
\hline
\end{tabular}


Por ambos motivos, es necesario analizar los resultados obtenidos de cada una de las velocidades del modelo, de las correcciones de estación y de la localización de sismos para entender y respaldar las nuevas determinaciones en relación a estudios geofísicos y geológicos existentes para la región.

\section{Modelo de Vp}

El modelo cortical de Vp final es en general similar al inicial, sin embargo leves diferencias podrían resultar significantes (Figura 6, Tabla 4). La velocidad de corteza superior resultó levemente inferior con una disminución de $0.07 \mathrm{~km} / \mathrm{s}$ respecto del valor original. Por el contrario, la velocidad de corteza media permaneció prácticamente inalterable. Llamativamente, la determinación de $\mathrm{Vp}$ de corteza inferior obtenida presenta una notable disminución de velocidad en relación al modelo inicial.

\section{Modelo de Vp de corteza superior}

La velocidad final de capa superior $(5.95 \mathrm{~km} / \mathrm{s})$ ha sido levemente mayor a la del modelo inicial $(5.88 \mathrm{~km} / \mathrm{s})$. Esta capa representa velocidades por encima de $10 \mathrm{~km}$ de profundidad hasta la elevación topográfica de cada estación. La región de estudio pertenece a la provincia estructural de Sierras Pampeanas que consiste en una serie de bloques de basamento ígneo-metamórfico levantados durante la orogenia Andina (Ramos et al., 2002; Rapela et al., 1998). Entre estos bloques se desarrollan una serie de depósitos neógenos que desarrollan el denominado sistema de cuencas de antepaís fragmentado (Jordan et al., 1986). La sierra de Pie de Palo, compuesta por diversas unidades metamórficas es un ejemplo de bloque de basamento elevado. Al sur de la sierra de Pie de Palo, un reprocesamiento sísmica profunda (línea L91017) reconoce una máxima profundidad de secuencias neógenas y preneógenas de $5 \mathrm{~km}$ en la cuenca de antepaís de Jocolí (Vergés et al., 2007; Comínguez y Ramos, 1991) y la manifestación morfológica de la Llanura Nororiental Mendocina, una planicie de gran extensión. Sobre el borde oeste del terreno Cuyania la unidad morfoestructural de precordillera expone rocas sedimentarias paleozoicas y mesozoicas. La red de estaciones se encuentra cubriendo principalmente el área de Cuyania donde se desarrolla esta cuenca de antepaís. En este contexto estructural, los sedimentos neógenos se encuentran en general por encima de las sedimentitas pre-neógenas y del basamento metamórfico. La recopilación global de velocidades para típicas rocas corticales muestran que valores por debajo $5.6 \mathrm{~km} / \mathrm{s}$ se corresponden a rocas sedimentarias, mientras que valores por encima de $5.7 \mathrm{~km} / \mathrm{s}$ corresponden a rocas ígneas o metamórficas (Brocher, 2005). Así mismo, las velocidades promedio globales indican que las rocas ígneas y metamórficas típicas presentan en general valo- 
res mayores a $5.7 \mathrm{~km} / \mathrm{s}$ (Christensen y Mooney, 1995). Se podría esperar que la importante participación de las velocidades sísmicas de sedimentos en los primeros $10 \mathrm{~km}$ de corteza superior fuera considerable y consecuentemente obtener un $\mathrm{Vp}$ de $\sim 5.6 \mathrm{~km} / \mathrm{s}$ o menor. Sin embargo, nuestra determinación es mayor inclusive al $\mathrm{Vp}$ inicial. Esto implica una mayor participación de un basamento metamórfico en la corteza superior. Altas velocidades de la corteza promedio de $\sim 6.4 \mathrm{~km} / \mathrm{s}$ fueron vinculadas a la asociación de rocas metamórficas de origen de arco islándico del terreno Cuyania (Alvarado et al., 2007). Similares valores también se obtuvieron en toda la corteza y fueron asociadas al efecto en profundidad de las rocas metamórficas que se exponen en la sierra de Pie de Palo (Castro de Machuca et al., 2010). Estas rocas vinculadas a las evidencias de altas velocidades han sido expuestas aisladamente al norte de la red. Esta tipos de rocas dentro de la zona de estudio junto con otras no expuestas y desconocidas podrían aumentar el promedio de $\mathrm{Vp}$ de corteza superior obtenido aquí.

\section{Corteza media}

La determinación final de $\mathrm{Vp}$ para la corteza media $(6.15 \mathrm{~km} / \mathrm{s})$ no ha presentado un cambio significativo con respecto al modelo de $\mathrm{Vp}$ inicial $(6.20 \mathrm{~km} / \mathrm{s})$. Además, existe mayoritaria consistencia de este valor con los considerados para el modelo de corteza promedio para localización de sismos (Anderson et al., 2007). Las determinaciones de $\mathrm{Vp}$ usando sensibilidad de Vp para inversión de forma de ondas regionales mostraron que la corteza promedio de Cuyania presenta sobre su franja este valores de 6 a $6.2 \mathrm{~km} / \mathrm{s}$ mientras que la franja oeste de 6.2 a $6.4 \mathrm{~km} / \mathrm{s}$ (Alvarado et $a l ., 2007)$. Estos antecedentes de Vp promedio de toda la corteza solo son consistentes con nuestra determinación de $\mathrm{Vp}$ para corteza media y que representa la capa de velocidad de mayor espesor de nuestro modelo. El modelo aquí obtenido más detallado en profundidad muestra valores de $\mathrm{Vp}$ para corteza superior e inferior que se alejan notablemente de estos valores de Vp promedio. Esta variabilidad de velocidades en profundidad pone de manifiesto la necesidad de considerar capas adicionales en un modelo unidimensional.

\section{Corteza inferior}

El valor de $\mathrm{Vp}$ final $(6.80 \mathrm{~km} / \mathrm{s})$ ha sido notablemente menor al sugerido por el modelo inicial $(7.3 \mathrm{~km} / \mathrm{s})$. Los modelos de velocidades promedio de corteza inferior considerando rayos comprendidos principalmente dentro de Cuyania y al norte de $31^{\circ} \mathrm{S}$ han determinado $\mathrm{Vp}>7.3 \mathrm{~km} / \mathrm{s}$ (Alvarado et al., 2007). Las funciones de receptor telesísmicas a $30^{\circ} \mathrm{S}$ han mostrado un débil contraste en la discontinuidad de Moho (Gilbert et al., 2006). Calckins et al., 2006 construyó funciones de recep- 
tor de sismos locales para estación sobre borde suroeste de Sierra de Pie de Palo y también ha sugerido la posibilidad de una transición "suave" de velocidad entre la corteza inferior y el manto subMoho. Estos autores coinciden en que las evidencias sismológicas se corresponden a una corteza inferior de alta velocidad y de composición parcialmente eclogitizada. Esto contrasta notablemente con nuestra determinación de $6.8 \mathrm{~km} / \mathrm{s}$. Sin embargo, las evidencias de eclogitización fueron obtenidas fundamentalmente al norte de $31^{\circ} \mathrm{S}$ dentro del terreno Cuyania. Las determinaciones de este estudio representan velocidades promedio de corteza inferior al sur de $31^{\circ} \mathrm{S}$. La velocidad obtenida aquí no se corresponde a la hipótesis de corteza inferior eclogitizada. Esto sugiere la posibilidad que el terreno Cuyania presenta una segmentación composicional en la corteza inferior a nivel regional.

\section{Manto superior}

La velocidad aquí obtenida para el manto superior fue de $7.4 \mathrm{~km} / \mathrm{s}$. Esta no es consistente con las velocidades encontradas al norte a $30^{\circ} \mathrm{S}$, donde valores de $8.2 \mathrm{~km} / \mathrm{s}$ fue calculado a partir de ondas refractadas Pn (Fromm et al., 2004). Además, esta se encuentra muy por debajo de la velocidad promedio global del modelo IASPEI91 para primeros $50 \mathrm{~km}$ de manto superior de $8.04 \mathrm{~km} / \mathrm{s}$ (Kennett, 1991). Esta determinación si es consistente con la anomalía de bajo $\mathrm{Vp}(\sim 7.6 \mathrm{~km} / \mathrm{s})$ del manto superior a profundidad de $85 \mathrm{~km}$ localizada por encima de la placa subducida donde se dispone plana (Wagner et al., 2005). Sin embargo, la red de estaciones CHARSME fue localizada sobre la región donde la placa subducida plana presenta un doblez para acomodarse a subducción normal al sur de $33.5^{\circ} \mathrm{S}$. Puede esperarse que los rayos provenientes de los sismos de profundidad intermedia no atraviesen en gran proporción el volumen anómalo de bajo Vp. Otra anomalía de bajo $\mathrm{Vp}(\sim 7.5 \mathrm{~km} / \mathrm{s})$ se encuentra a $65 \mathrm{~km}$ de profundidad entre latitudes 32.5 y $33.5^{\circ} \mathrm{S}$ y longitudes 70 y $70.7^{\circ} \mathrm{O}$ (Wagner et al., 2005). Su volumen reducido y localización sobre el borde de la red cuestionan su influencia sobre nuestra determinación. Otra anomalía de alto $\mathrm{Vp}(8.4 \mathrm{~km} / \mathrm{s})$ centrada a $85 \mathrm{~km}$ y en la zona de transición de placa plana a normal y comprendida dentro de la red no es consistente con nuestro resultado. Es posible considerar que un efecto combinado debido a la participación relativa de las heterogeneidades de $\mathrm{Vp}$ de manto superior en los tiempos de viaje de sismos seleccionados sea la causa de esta determinación de bajo Vp. Aún bajo este supuesto el Vp determinado aquí es aún más bajo que cualquier anomalía de menor Vp determinada por Wagner et al., 2005. Así mismo, el promedio aritmético de las velocidades de las tres anomalías de $7.8 \mathrm{~km} / \mathrm{s}$ es mayor al valor de $\mathrm{Vp}$ aquí obtenido.

La capa de velocidad de manto superior, al contrario de las capas del modelo de $\mathrm{Vp}$ de corteza, esta solamente controlada por los rayos que parten de los sismos de profundidad intermedia y arriban a las estaciones sismológicas. La cantidad de 
sismos involucrados en el proceso de inversión fue de 72 y tan solo representa el $30 \%$ del total de los sismos involucrados. Una buena determinación de velocidad considera un número suficiente de rayos cuyas trayectorias se entrecrucen y muestreen un volumen en diversos sentidos y direcciones. Se puede considerar que la cantidad de sismos involucrados no es la suficiente. Así mismo se puede sugerir que los rayos que partieron de los sismos principalmente agrupados sobre la zona noroeste no describan el suficiente entrecruzamiento y la variedad de trayectorias para condicionar esta gruesa capa de Vp de manto superior. Si esto es cierto, la velocidad para manto superior sería pobremente condicionada y, en ese caso, la determinación no sería confiable.

Se probó el desempeño del modelo de $\mathrm{Vp}$ definiendo la velocidad de manto superior a $8.04 \mathrm{~km} / \mathrm{s}$ que es el valor promedio del modelo global de velocidades ISPEI91 (Kennett, 1991). La relocalización de sismos mediante el método JHD (se mantuvo invariable el modelo de Vp obtenido de corteza y el de manto superior) indicó un RMS de $0.315 \mathrm{~s}$. Este valor fue muy similar al obtenido de $0.311 \mathrm{~s}$ con Vp de manto de $7.4 \mathrm{~km} / \mathrm{s}$. El cambio débil cambio de $1.3 \%$ entre ambos modelos de manto superior fue interpretado como el poco control de los rayos provenientes de los sismos de placa en el condicionamiento de la determinación de $\mathrm{Vp}$ del manto superior. Además, también indica la relativa poca participación de lectura de arribos de los sismos de profundidad intermedia en el RMS total del proceso de inversión que integra también a los sismos de corteza. Se puede sugerir que los sismos de profundidad intermedia no condicionan lo suficiente la determinación de Vp de manto superior.

En esta situación de determinación probablemente mal condicionada, se definió al modelo de $\mathrm{Vp}$ de manto superior con el valor de $8.04 \mathrm{~km} / \mathrm{s}$. Este valor fue usado como el inicial para obtener modelos tomográficos por Wagner et al., 2005. Por otro lado, el modelo unidimensional más adecuado como inicial para obtener el más representativo modelo tomográfico tridimensional es denominado modelo mínimo de velocidades (Kissling et al., 1994). Así, se puede interpretar que el modelo unidimensional inicial de Wagner et al., 2005 con el valor inicial de $8.04 \mathrm{~km} / \mathrm{s}$ ha sido considerado implícitamente como modelo mínimo. Esto refuerza la elección del valor de Vp de IASPEI91 por sobre otras velocidades posibles del manto superior.

\section{Correcciones de estación}

Las correcciones de estación permiten compensar los efectos sistemáticos de residuos de tiempos de viaje asociados generalmente a heterogeneidades de velocidades que no pueden ser adecuadamente resueltas por el modelo unidimensional. Para las estaciones con uniforme distribución azimutal y ángulos de incidencia de rayos, las correcciones de estación reflejan heterogeneidades de subsuperficie cercana. Para 
las estaciones cercanas al borde de la red la cobertura de rayos es limitada y correcciones de estación representan tanto los efectos de anomalías de subsuperficie cercana como los efectos sistemáticos producidos por anomalías de velocidad más profundas. AGRE ha sido seleccionada como la estación de referencia en base al conocimiento geológico de la zona. Los retardos por arriba o por debajo representan, bajo las condiciones antes descriptas, velocidades menores o mayores que las presentes en subsuperficie de estación AGRE.

La mayoría de los valores de corrección de estación se encuentran comprendidos entre -0.1 a 0.2 segundos (Figura 7). Este rango de valores se encuentra en el orden de valores de 0.2 a $-0.5 \mathrm{~s}$ para las correcciones de estación alrededor de la sierra de Pie de Palo obtenidas por una red de estaciones temporaria (Pujol et al., 1992). Este autor ha obtenido valores de retardos negativos en promedio de -0.4 en una franja comprendida entre la unidad morfotectónica de Precordillera Oriental y la sierra de Pie de Palo que son completamente consistentes con el retardo de $-0.39 \mathrm{~s}$ para la estación CIEN localizada sobre Precordillera Oriental. Esta correspondencia confirma la compatibilidad de nuestros datos y resultados con otras investigaciones aun cuando estas fueron realizadas considerando datos de otra red de estaciones.

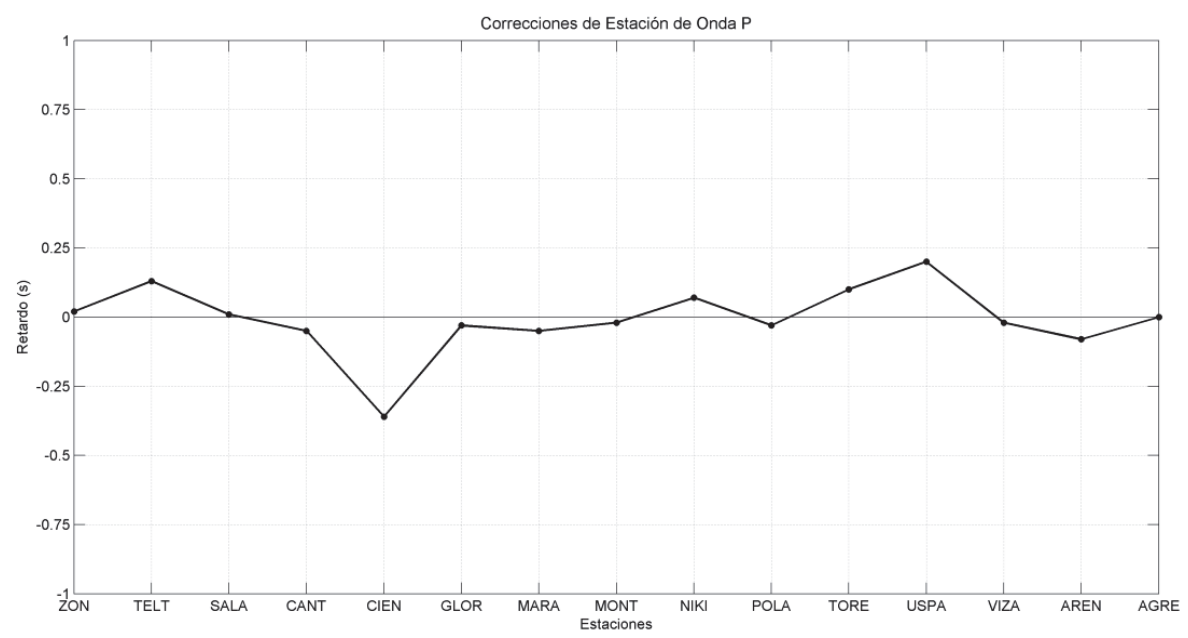

Figura 7. Valores de corrección de estación para cada estación sismológica.

\section{Desempeño final}

Se denominó modelo unidimensional mejorado de $\mathrm{Vp}$ al modelo formado por las velocidades de corteza obtenidas mediante el proceso de inversión junto al modelo de $\mathrm{Vp}$ de manto superior de $8.04 \mathrm{~km} / \mathrm{s}$ (Figura 8). El nuevo modelo de velocidades 
con sus correspondientes correcciones de estación disminuyó el RMS en un 20\% en comparación con las localizaciones iniciales. Como las correcciones de estación compensan las desviaciones sistemáticas de residuos, la disminución de RMS no es un efecto únicamente relacionado con el modelo mejorado de $\mathrm{Vp}$. Considerando el modelo mejorado y la relocalización sin ajuste de corrección de estación el RMS disminuyó a $0.335 \mathrm{~s}$. Esta disminución indica la ventaja de presentar un modelo de $\mathrm{Vp}$ adecuadamente construido para una región determinada.

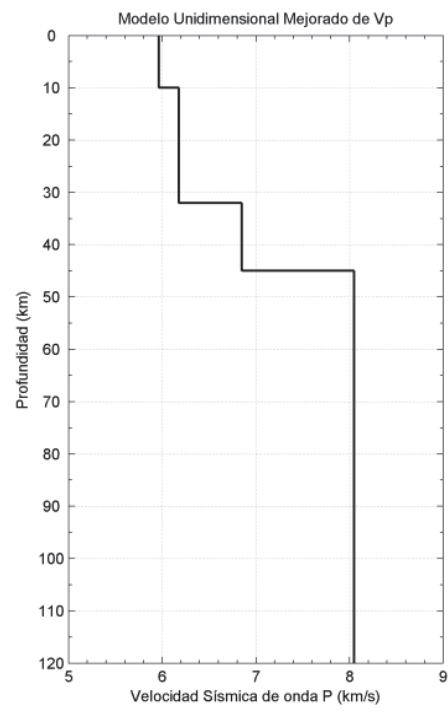

Figura 8. Modelo unidimensional mejorado de $\mathrm{Vp}$ con velocidad de manto superior correspondiente al promedio global de velocidades del modelo IASPEI 91.

El desempeño para el total de sismos detectados (fuera y dentro de la red) también muestra una disminución de indicadores de desempeño. El RMS total para 449 sismos de corteza y 1,262 sismos de profundidad intermedia disminuyó de 0.84 a 0.80. La disminución significa que el modelo de Vp mejorado también es más adecuado para una región afuera de la red de estaciones. Sin embargo, esta disminución es relativamente menor al $20 \%$ obtenida considerando solo los sismos seleccionados. Si bien el modelo mejorado aumentó su desempeño, este no lo es en la cantidad obtenida para sismos seleccionados lo que indica que a pesar que el modelo mejorado presenta mayor generalidad y es más adecuado y representativo para el espacio de velocidades local dentro de la red que una zona más regional circundante, este es aún más representativo de las velocidades en profundidad de la región dentro de la red de estaciones. 
La nueva localización de hipocentros muestra que la distribución de sismos en el periíodo reducido de tres meses (Figura 9 y 10) se corresponden espacialmente con sismicidad histórica conocida mediante catálogos internacionales de los últimos 20 años (United States Geological Survey -USGS, National Earthquake Information Center-NEIC) (Figura 1). Las localizaciones finales muestran un patrón de sismicidad de corteza y asociada a placa subducida que manifiestan nuevos detalles de agrupamientos y lineamientos de sismos no vistos antes para la región. La sismicidad de corteza se localizó fundamentalmente dentro del terreno Cuyania o bien en el límite entre este y terrenos adyacentes. Esta evidencia es completamente consistente con la afirmación de Alvarado et al., 2007 donde la máxima liberación de energía elástica se encuentra dentro de este terreno. Por otro lado, los estudios previos de localizaciones de sismos asociados a placa subducida de Nazca (Anderson et al., 2006; Wagner et al., 2005) muestran un patrón general de sismicidad que coincide con nuestras localizaciones. Particularmente interesante son los sismos de placa de Nazca que se muestran concentrados en grupos o lineamientos no antes conocidos (Anderson et al., 2007). Sin embargo, el análisis detallado de la sismicidad a partir de las localizaciones de menor error escapa el ámbito de este trabajo.

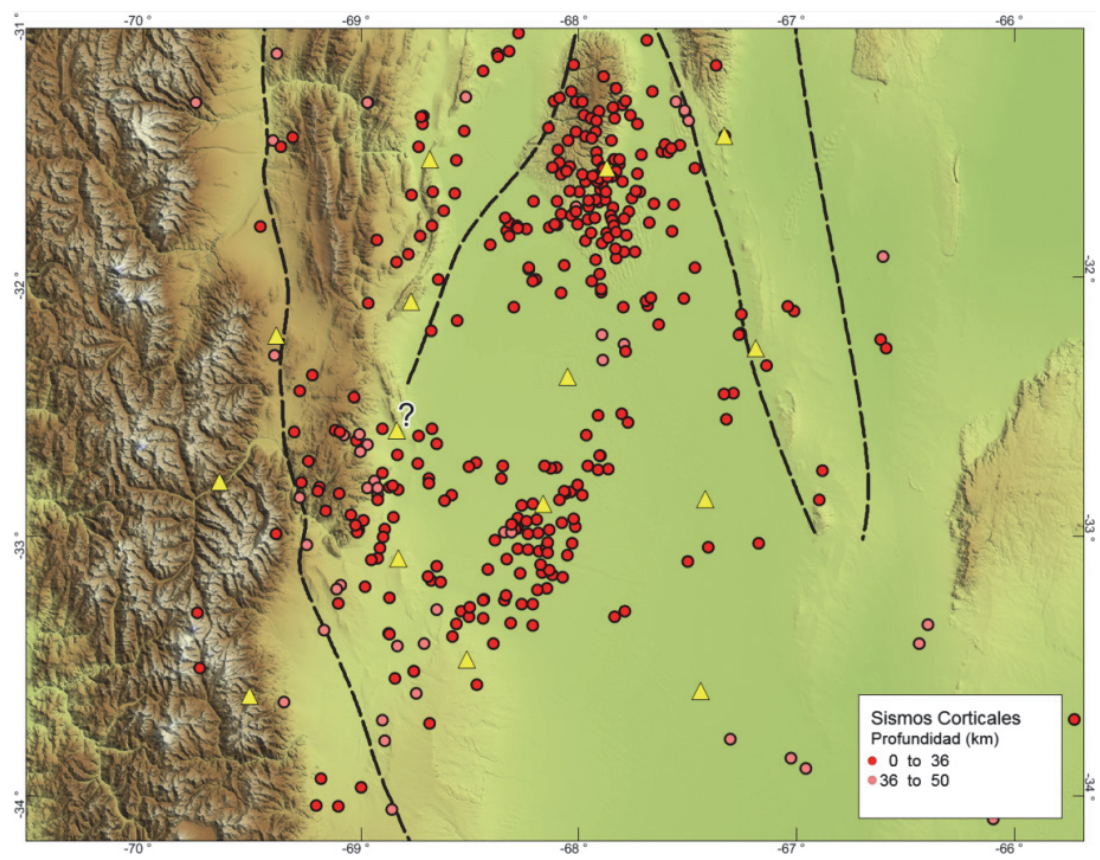

Figura 9. Sismos de corteza identificados durante el experimento CHARSME y relocalizados usando el modelo mejorado de Vp. 


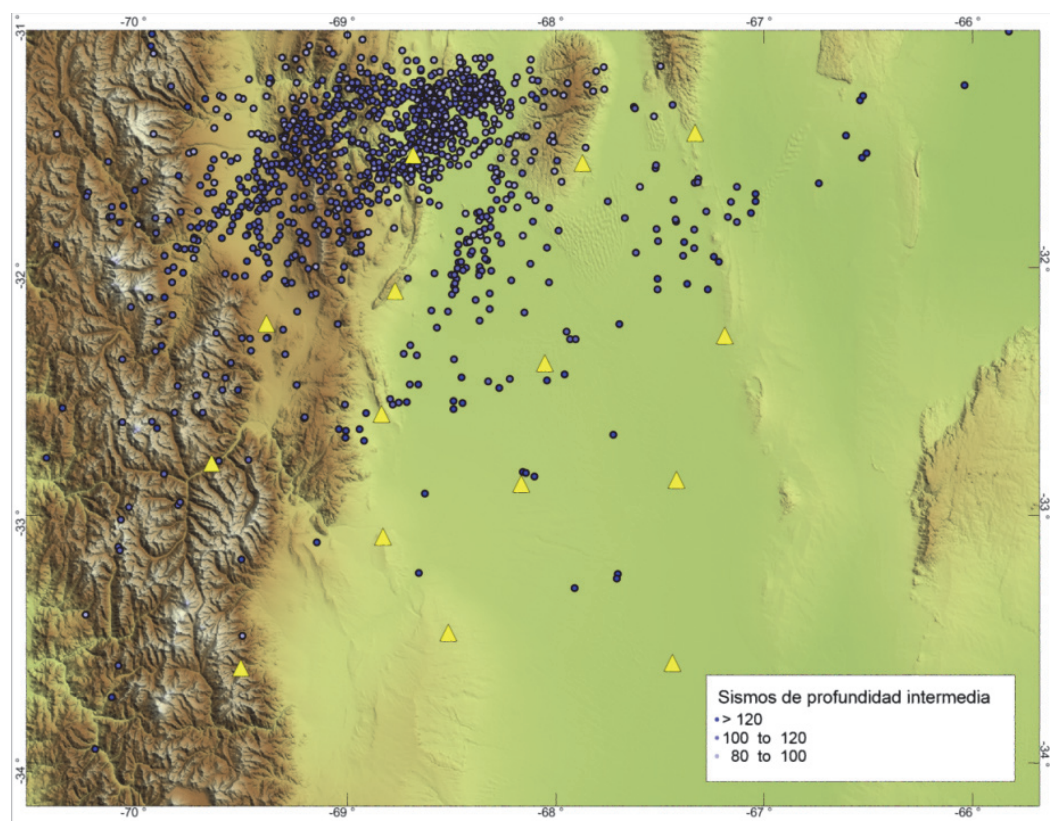

Figura 10. Sismos de profundidad intermedia detectados y relocalizados usando nuevo modelo de Vp. La mayoría de estos sismos se localizada fuera del área de cobertura de la red.

\section{Conclusiones}

Se obtuvo un nuevo y mejorado modelo de velocidades de cuatro capas a partir de nuevos datos sismológicos de una red local que constituye el único modelo unidimensional de velocidades de corteza y manto superior para la zona que fue obtenido a partir de la inversión de primeros arribos de ondas $\mathrm{P}$ de sismos de corteza y profundidad intermedia.

Se analizaron cuidadosamente los parámetros que controlan el proceso de inversión. Todas las determinaciones de Vp del modelo obtenido han sido validadas con antecedentes geofísicos y geológicos. La velocidad de la corteza superior mayor a la del modelo inicial sugiere una mayor participación de rocas de basamento metamórfico de altas velocidades, algunas expuestas principalmente en la sierra de Pie de Palo y otras no expuestas y desconocidas. La corteza media presenta una velocidad similar a antecedentes de velocidades promedio para toda la corteza indicando la necesidad de considerar modelos de mayor cantidad de capas en profundidad. La velocidad obtenida para la corteza inferior fue menor a la esperada y conocida dentro del terreno Cuyania lo que implica una posible segmentación de la composición 
a este nivel cortical, parcialmente eclogitizada al norte de la sierra Pie de Palo pero que no parece tener las mismas características al sur. El Vp obtenido de manto superior mal condicionado fue definido al valor de $8.04 \mathrm{~km} / \mathrm{s}$ sin notable aumento del RMS. Las correcciones de estación demuestran la consistencia de nuestras determinaciones de subsuperficie somera en relación a las determinadas por otros experimentos sismológicos. La disminución de RMS en un 20\% implica un menor error de localización y mayor adecuación de nuestro modelo de $\mathrm{Vp}$ de corteza y manto superior y permitirá en futuros trabajos reconocer nuevas características de la sismicidad de corteza y placa subducida.

Este mejorado modelo se propone como uno adecuado para una rápida y relativamente precisa relocalización tanto para sismos de corteza comprendidos fundamentalmente dentro de Cuyania como de profundidad intermedia asociados a la placa subducida de Nazca, en la zona de transarco andino argentino entre $31.5 \mathrm{y}$ $33.5^{\circ} \mathrm{S}$ donde se produce la mayor actividad sísmica en niveles someros y a profundidad intermedia.

El modelo mejorado de Vp podrá ser usado como inicial para obtener otro modelo de velocidades más detallado en profundidad y que no solo sea de onda $\mathrm{P}$, sino también de onda $\mathrm{S}$ y de relación $\mathrm{Vp} / \mathrm{Vs}$. Este conjuntamente con otros modelos tentativos iniciales podrán ser considerados para obtener un modelo mínimo unidimensional de velocidades de Vp que como tal, permita no solo obtener localizaciones más precisas de hipocentros y ser usado como modelo inicial para construcción de modelos tomográficos de sismos locales, si no también y así mismo proveer interpretaciones preliminares sobre la estructura en velocidad de los niveles profundos de la Tierra y su correspondencia con composición y petrología.

\section{Referencias}

Aceñolaza, F.G.; Miller, H. and Toselli, A.J., 2002. Proterozoic-Early Paleozoic evolution in western South America, a discussion. Tectonophysics, 354, 121-137 Alvarado, P.; Beck, S. and Zandt, G., 2007. Crustal structure of the south-central Andes Cordillera and backarc region from regional waveform modelling. Geophysics. J. Int.

Anderson, M.; Alvarado P.; Zandt, G. and Beck, S., 2007. Geometry and brittle deformation of the subducting Nazca Plate, Central Chile and Argentina. Geophysical Journal International, 171(1), 419-434.

Bollinger, G.A. and Langer, C.J., 1988. Development of a velocity model for locating aftershocks in the Sierra Pie de Palo region of western Argentina, USGS Bull. 1795

Castro de Machuca, B. y Perarnau, M., 2012. A seismological and petrological crustal model for the Southwest of the Sierra de Pie de Palo, province of San Juan. Revista de la Asociación Geológica Argentina, 69(2), 177-184. 
Brocher, T., 2005. Empirical relations between elastic wavespeeds and density in the earth's crust. Bull. Seismol. Soc. Am., 95(6), 2081-2092.

Cahill, T. and Isacks, B., 1992. Seismicity and shape of the subducted Nazca plate. J. Geophys. Res., 97, 17 503-17 529.

Calkins, J.A.; Zandt, G.; Gilbert, H.J. and Beck, S.L., 2006. Crustal images from San Juan, Argentina, obtained using high frequency local event receiver functions, Geophys. Res. Lett., 33.

Christensen N.I. and Mooney W.D., 1995. Seismic velocity structure and composition of the continental crust: a global view, J. Geophys. Res., 100, 9761-9788,

Eberhart-Phillips D., and Michael A.J., 1993. Three-dimensional velocity structure, seismicity, and fault structure in the Parkfield Region, central California. J. Geophys. Res., 98(B9), 15737-15758.

Fromm, R.; Zandt, G. and Beck S.L., 2004. Crustal thickness beneath the Andes and Sierras Pampeanas at $30 \circ \mathrm{S}$ inferred from Pn apparent phase velocities, Geophys. Res. Lett., 31.

Gilbert, H.; Beck, S. and Zandt G., 2006. Lithosperic and upper mantle structure of Central Chile and Argentina, Geophys. J. Int., vol. 165, pp. 383-398. Goldstein, P., Dodge, D.\&Firpo, M., 1999.

Gutscher, M.A., 2002. Andean Subduction Styles and their Effect on Thermal Structure and Interplate Coupling. Journal of South American Earth Sciences, 15, 3-10.

Heit, B.; Yuan, X.; Bianchi, M.; Sodoud, F. and Kind R., 2008. Crustal thickness estimation beneath the southern Central Andes at 30 degrees S and 36 degrees S from $\mathrm{S}$ wave receiver function analysis, Geophysical Journal International, 74(1), 249-254.

Hildreth, W.; Singer, B.; Godoy, E. and Munizaga, F., 1998. The age and constitution of Cerro Campanario, a mafic stratovolcano in the Andes of central Chile. Revista Geologica de Chile, 2, 17-28.

Husen, S.; Kissling, E. and Quintero, R., 2002. Tomographic evidence for a subducted seamount beneath the Gulf of Nicoya, Costa Rica: The cause of the 1990 $\mathrm{Mw}=7.0$ Gulf of Nicoya earthquake. Geophys. Res. Lett., 29(8).

Ji, S.C.; Wang, Q. and Xia, B., 2002. Handbook of Seismic Properties of Minerals, Rocks and Ores. Polytechnic International Press, Montreal, Polytechnic Int., Montreal, Quebec, Canada, 630 pp.

Jordan, T.E. and Allmendinger R.W., 1986. The Sierras Pampeanas of Argentina: a modern analogue of Rocky Mountain foreland deformation. Am. J. Sci., 286, 737-764.

Kay, S. Mahlburg and C. Mpodozis, 2002. Magmatism as a probe to the Neogene shallowing of the Nazca plate beneath the modern Chilean flatslab. Journal of South American Earth Science, 15, 39-59.

Kennett B.L.N., and Engdahl E. R., 1991. Travel times for global earthquake location and phase identification. Geophys. J. Int., 105, 429-466. 
Kissling, E., 1988. Geotomography with local earthquake data. Rev. Geophys., 26, 659-698,

Kissling, E.; Ellsworth, W.L.; Eberhart-Phillips, D. and Kradolfer U., 1994. Initial reference models in local earthquake tomography. J. Geophys. Res., 99.

Kissling, E.; Kradolfer, U. and Maurer, H., 1995. VELEST user's guide-short introduction, Institute of Geophysics and Swiss Seismological Service, ETH, Zurich, 25 pp.

Klein, F., 2002. User's Guide to HYPOINVERSE-2000: A Fortran Program to Solve for Earthquake Locations and Magnitudes[R], USGS Open File Report Ver1. 0.

McGlashan, N.; Brown, L. and Kay, S., 2008. Crustal thickness in the Central Andes from teleseismically recorded depth phase precursors. Geophysical Journal International, 175(3), 1013-1022.

Perarnau, M.; Alvarado P. and Saez, M., 2010. Estimación de la estructura cortical de velocidades sísmicas en el suroeste de la Sierra de Pie de Palo, provincia de San Juan (Argentina). Revista de la Asociacion Geologica Argentina, 67.

Pujol, J., 1992. Joint hypocentral location in media with lateral velocity variations and interpretation of the station corrections. Physics of Earth and Planetary Interiors, 75, 7-24.

Ramos, V.A.; T.E., Jordan; R.W. Allmendinger; C., Mpodozis; S.M., Kay; J.M. Cortés, and M. Palma, 1986. Paleozoic terranes of the central Argentine-Chilean Andes, Tectonics, 5(6), 855-880.

Ramos, V.A.; Cristallini, E.O. and Pérez, D.J., 2002. The Pampean flat-slab of the central Andes. J. South Am. Earth Sci., 15, 59-78.

Ramos, V.A., 2009. The Grenville-Age Basement of the Andes. Journal of South American Earth Sciences.

Volponi, F., 1968. The earthquakes of Mendoza of 21 October 1968 and the structure of the earth's crust. Acta Cuyana de Ingeniería, 12, 95-101.

Smalley R. Jr.; Pujol, J.; Regnier, M.; Chiu, J.M.; Chate Iain, J.L.; Isacks, B.L.; Araujo, M. and Puebla, N., 1993. Basement seismicity beneath the Andean Precordillera thin-skinned thrust belt and implications for crustal and lithospheric behavior. Tectonics, 12, 63-76. 